\title{
Acceso abierto, promoción y difusión digital de proyectos I+D+i: el caso de la Universidad Complutense de Madrid
}

\author{
Open access, promotion and digital dissemination of $R+D$ projects: the case of the Complutense University of Madrid
}

\section{Brenda SISO-CALVo, Rosario ARQUERO-AvILÉS}

\begin{abstract}
Universidad Complutense de Madrid, Departamento de Biblioteconomía y Documentación, Grupo de Investigación
\end{abstract} IDEA_LAB, C/ Santísima Trinidad, 37. 28010 Madrid (España), msiso@ucm.es, carquero@ucm.es

\begin{abstract}
Resumen
Se analizan las estrategias y planes de difusión digital de los proyectos de investigación de la convocatoria 2013 del Plan Estatal de Investigación Científica y Técnica concedidos a la Universidad Complutense de Madrid (UCM) en relación con el acceso abierto. Se recuperó la producción científica de los 55 proyectos seleccionados en las bases de datos Web of Science y Scopus, el repositorio institucional de la UCM (E-prints Complutense) y el buscador Google Scholar. De los 429 documentos recuperados se analiza la producción en acceso abierto, el grado de cumplimiento de las políticas nacionales e institucionales sobre acceso abierto, la producción por ramas de conocimiento, y la existencia de páginas web específicas. Entre los resultados destaca la ausencia de estrategias o planes de difusión y promoción de la investigación, así como el escaso cumplimiento de la política institucional sobre acceso abierto.
\end{abstract}

Palabras clave: Proyectos I+D+i. Acceso abierto. Difusión de la investigación. Promoción de la investigación. Comunicación académica. Universidad Complutense de Madrid.

\section{Introducción}

\subsection{Los proyectos I+D+i en el contexto} universitario

La investigación, como función esencial del Sistema Universitario Español, viene recogida en la diversa normativa como la Ley Orgánica 6/2001, de 21 de diciembre, de Universidades (LOU) y la Ley 14/2011, de 1 de junio, que regula el actual contexto del Sistema Español de Ciencia, Tecnología e Innovación.

La LOU profundiza en los aspectos relacionados con el desempeño de la investigación en el ámbito universitario y dedica expresamente su Título VII a la investigación y transferencia de conocimiento, poniendo de manifiesto la importancia de la investigación como factor competitivo para las universidades (España, 2001). El fomento y promoción de la investigación científica y técnica

\begin{abstract}
The strategies and plans for digital dissemination of the research projects of the 2013 call for proposals of the State Plan for Scientific and Technical Research granted to the Complutense University of Madrid (UCM) are analysed in relation to open access. The scientific production of the 55 selected projects was recovered from the Web of Science, Scopus, Institutional Repository of the UCM (E-prints Complutense) and Google Scholar. Of the 429 documents retrieved, open access production, the degree of compliance with national and institutional policies on open access and production by discipline were analysed. In relation to the dissemination of information on the projects, the existence of specific web pages was analysed. Among the main results the lack of strategies or plans for the dissemination and promotion of research, as well as the lack of compliance with the institutional policy on open access should be outlined.
\end{abstract}

Keywords: R+D projects. Open access. Research dissemination. Research promotion. Scholarly communication. Complutense University of Madrid.

viene establecido en la LOU con el fin de contribuir a la generación, difusión y transferencia del conocimiento. Concretamente, el artículo 41 determina como aspecto clave la promoción de sistemas innovadores de organización y gestión de la actividad investigadora, así como del fomento y la transferencia de los resultados de la investigación (España, 2001).

Los distintos gobiernos han contribuido a la transformación de la investigación universitaria a través del establecimiento de políticas científicas que han permitido a las universidades desarrollar su función investigadora mediante la disponibilidad de financiación pública para la investigación, desarrollo e innovación (I+D+i). Por tanto, la relevancia de la I+D+i en la vida de la universidad ha sido introducida mediante la organización de mecanismos de financiación, principalmente competitivos y por medio del desarrollo de proyectos I+D+i (Sanz Menéndez, 2005). 
El mecanismo principal de financiación corresponde al Plan Estatal de Investigación Científica y Técnica, que permite la estabilidad de financiación anual basada en proyectos. Dicho Plan está contemplado en el artículo 42 de la Ley de la Ciencia, la Tecnología y la Innovación con el objetivo de financiar las actuaciones en materia de investigación científica y técnica que se correspondan con las prioridades establecidas por la Administración General del Estado, y orientar las actividades de $\mathrm{I}+\mathrm{D}+\mathrm{i}$ hacia los retos de la sociedad para la producción de bienes y servicios que promuevan la generación de ventajas competitivas (España, 2011). El Plan Estatal de Investigación Científica y Técnica y de Innovación para el periodo 2017-2020 constituye, junto con el anterior Plan Estatal correspondiente al período 2013-2016, el instrumento fundamental de la Administración General del Estado para el desarrollo y consecución de los objetivos estratégicos de la Estrategia española de Ciencia, Tecnología e Innovación 2013-2020.

\subsection{Los planes de difusión de la investigación}

Los aspectos relacionados con la difusión de resultados de investigación cobran especial relevancia en el contexto de los proyectos de investigación. Como ejemplo, y en consonancia con las políticas europeas para la financiación de proyectos de investigación, las bases reguladoras para la concesión de ayudas públicas del Programa Orientado a los Retos de la Sociedad en el marco del anterior Plan Estatal de Investigación Científica y Técnica y de Innovación 20132016 incluyen entre sus criterios de evaluación los planes de difusión contemplados en las propuestas presentadas (España, 2013), por lo que contar con buenas estrategias para la difusión de la investigación, principalmente en el contexto digital, se convierte en un aspecto clave a la hora de conseguir financiación.

La orientación de la investigación basada en proyectos, así como la importancia de la fase final del proceso de investigación científica relativa a la difusión digital de los resultados, hace imprescindible el conocimiento por parte de los académicos de técnicas de gestión de proyectos de investigación $\mathrm{y}$, concretamente, de las actividades relacionadas con la comunicación, promoción y difusión digital de los resultados del proyecto.

Como decíamos, la importancia de la formulación de planes de difusión se materializó en el Plan Estatal. Pero es importante advertir que las decisiones en el contexto nacional se rigen por las normas comunitarias sobre la materia, en consonancia con el esquema de la Unión Europea reflejado en el Programa Marco de Investigación,
Desarrollo e Innovación denominado Horizonte 2020. "La difusión de resultados del proyecto es considerada por la Comisión Europea como una de las actividades más importantes del mismo y debe iniciarse desde la primera fase del proyecto" (MINECO, 2014: 87).

El objetivo clave de un plan de difusión es transferir el conocimiento útil y utilizable a todas las partes interesadas, incluidos la comunidad investigadora, los profesionales, la sociedad, etc., por lo que resulta fundamental la planificación de estrategias adecuadas que evidencien que los resultados se orientan a las necesidades de los usuarios y/o beneficiarios finales (Fundación Botín, 2016).

La importancia de la difusión en los proyectos de investigación descansa en la propia naturaleza de la ciencia. López Yepes (2010) determina que la ciencia es un proceso de comunicación mediante el que el productor de la investigación científica permite la difusión de los resultados para que un usuario o nuevo investigador pueda aprovechar el contenido como fuente para la realización de su trabajo, que también se difundirá, y así sucesivamente en una cadena sin fin. Al mismo tiempo, el conocimiento científico exige publicidad, pues un descubrimiento no se da por concluido realmente hasta que las partes interesadas tienen constancia.

En esta misma línea, para Seguí Simarro, Poza Luján y Mulet Salort (2015) es esencial realizar un correcto marketing científico. Entre los motivos que avalan dicha afirmación, los autores aluden a aspectos éticos, para comunicar a la sociedad en qué se han gastado su dinero y poner en relevancia frente a los políticos que el presupuesto para investigación es una inversión; y a aspecto prácticos, para poner en valor el trabajo de los propios investigadores con el fin de mejorar la imagen pública y lograr más visibilidad, reconocimiento y reputación académica.

La demostración de que el contexto digital se configura como un escenario óptimo para la promoción y difusión de la investigación corresponde al surgimiento y uso de plataformas específicas como las redes sociales académicas (Mendeley, ResearchGate, Academia.edu. etc.), plataformas de identidades (Orcid, ResearcherID, etc.) y la aparición de movimientos relacionados con la aplicación de la web social a la investigación, como la ciencia abierta, ciencia 2.0 o las altmétricas, para medir el impacto y la visibilidad de la investigación en el entorno digital (Siso Calvo y Arquero Avilés, 2017).

En cuanto a su utilidad, la literatura coincide en que estas herramientas digitales permiten duplicar el acceso a las investigaciones y reducir el tiempo de retorno de las citas (Madisch, 2015 citado en 
Campos-Freire; Rúas-Araújo, 2016), comunicarse directamente con sus audiencias (López-Pérez; Olvera-Lobo, 2016), compartir documentos y proyectos (Campos-Freire, 2013 citado en GonzálezDíaz; Iglesias-García; Codina, 2016), así como multiplicar el número de citas (Lawrence, $2001 \mathrm{ci}-$ tado en García-Peñalvo, 2017). Según Abadal (2004), la creación de un sitio web dedicado es muy habitual debido a su gran alcance y posibilidad de ofrecer información actualizada.

\subsection{Ciencia abierta y acceso abierto}

La publicación académica, en concreto las revistas y monografías, que constituyen el núcleo de la comunicación académica, se ha enfrentado a una serie de desafíos y dificultades a lo largo de las últimas décadas derivados principalmente de los precios de suscripción de las revistas científicas (Baughman, 2015) y por la transformación de las prácticas de la comunicación académica debido a la revolución de las TIC e Internet (Sawant, 2012).

Suber (2015) afirma que el actual sistema de difusión de la investigación en revistas científicas es inadecuado para los investigadores y sus instituciones, pero muy rentable para las grandes editoriales convencionales. Los investigadores ceden sus trabajos a las revistas, de manera gratuita o incluso pagando algún tipo de tasa de publicación y, posteriormente, se debe pagar para poder acceder al contenido.

El acceso abierto a la ciencia, denominado Ciencia Abierta (Open Science), reclama el dominio púbico para la ciencia, permitiendo su difusión y reutilización y, por consiguiente, un rápido progreso científico y cultural. Supone, por tanto, un cambio de modelo y la instauración de un nuevo paradigma en el funcionamiento de la comunicación científica (Ollé y Abadal, 2013).

El acceso abierto, principal característica de la Ciencia Abierta, responde a las características propias del sistema, basado principalmente en la financiación pública, y en el interés de los propios investigadores en difundir los contenidos para un intercambio de conocimiento, potenciar su reputación $\mathrm{y}$, consecuentemente, obtener méritos profesionales para su promoción y ascenso en la carrera profesional (Suber, 2012).

Para Suber, "la literatura en acceso abierto (AA) es digital, online, gratuita y libre de restricciones de copyright y licencias de uso" (2012: 4).

Abadal (2014) clasifica esta doble vertiente del acceso abierto en económica, de forma que se permita el acceso gratuito a la producción científica, y legal, mediante la disposición de los contenidos libres de derechos de explotación.
En la Declaración de Budapest (Budapest Open Access Initiative, 2002) se establecieron dos vías, denominadas rutas, para alcanzar el acceso abierto, y son las que se han ido consolidando hasta la actualidad: la ruta verde y la ruta dorada.

Se conoce como la ruta dorada cuando el acceso se facilita a través de la publicación en revistas mediante la opción de acceso abierto, y la ruta verde cuando el autor deposita sus trabajos, incluidos los publicados en revistas convencionales, en repositorios institucionales o temáticos facilitando el acceso y consulta a través de estos.

Suber (2012) argumenta que la mayoría de los editores de revistas convencionales por suscripción recogen en su política el acceso abierto verde y otras lo conceden previa petición, existiendo cierta obligación por parte de las revistas cuando los autores actúan bajo imposición de las entidades o universidades que financian su investigación.

Por tanto, los principales agentes para la transformación e impulso de la ruta verde del acceso abierto corresponden a las universidades y organismos de financiación a través de sus políticas y su correcta formulación, instigando a las editoriales a que se adapten a sus criterios.

Esta situación actual, en la que la mayoría de las revistas convencionales conceden permisos para el depósito de los trabajos en repositorios, es uno de los triunfos más importantes del movimiento acceso abierto, por lo que la responsabilidad de aprovechar esa oportunidad corresponde a los investigadores.

\subsection{Iniciativas a favor del acceso abierto}

A nivel europeo, una de las iniciativas más influyentes se configura con el programa Horizonte 2020. Este programa, que financia proyectos de investigación, incluye entre las obligaciones de sus beneficiarios la de depositar en abierto todas aquellas publicaciones que se generen en el marco de los proyectos financiados (Parlamento Europeo, 2013).

En España, la Ley 14/2011, de 1 de junio, de la Ciencia, la Tecnología y la Innovación, recoge en su artículo 37 la difusión en acceso abierto, obligando al personal investigador a depositar en repositorios de acceso abierto (España, 2011, p. 54425):

El personal de investigación cuya actividad investigadora esté financiada mayoritariamente con fondos de los Presupuestos Generales del Estado hará pública una versión digital de la versión final de los contenidos que le hayan sido aceptados para publicación en publicaciones de investigación seriadas o 
periódicas, tan pronto como resulte posible, pero no más tarde de doce meses después de la fecha oficial de publicación.

La obligación legal de depositar en abierto supuso la creación de una comisión de seguimiento que midiese el grado de cumplimiento del mandato de depósito en abierto por parte de la comunidad investigadora.

El informe publicado por la comisión, resultado de una metodología compleja dada la difícil tarea de poder localizar los artículos fruto de proyectos de investigación nacionales, arrojó los primeros datos acerca de la situación del depósito en repositorios (ruta verde). Según los datos, el $9 \%$ de los artículos de proyectos de I+D+i nacionales financiados en el periodo 2012-2014 contenían alguna información sobre la financiación y se encontraban depositados en abierto en repositorios institucionales (FECYT, 2016).

Otro estudio al respecto es el realizado por Borrego (2016), que analiza 818 artículos publicados durante el 2012 en el marco de 299 proyectos financiados para el periodo 2012-2014. Los resultados demuestran que se obtuvo al menos una copia en acceso abierto de 478 artículos sobre los 818 artículos del conjunto de datos $(58,4 \%)$. En el propio estudio se advierte que la discrepancia entre los resultados de la diversa literatura radica en la metodología propuesta.

Otra de las iniciativas españolas corresponde al nuevo Plan Estatal Investigación Científica y Técnica y de Innovación 2017-2020, que promueve un modelo de I+D+i abierto y responsable apoyado en la participación de la sociedad, mediante el acceso abierto a resultados y datos de la investigación, así como impulsar un modelo de investigación responsable y abierta a la sociedad y el reconocimiento de los trabajospublicadosenacceso abiertoenrepositoriosinstitucionalesytemáticoscomopartede la actividad investigadora (MINECO, 2017).

En el contexto universitario, una de las declaraciones más importantes fue la propuesta por la Conferencia de Rectores de las Universidades Españolas (CRUE, 2004), aprobada por la XII Asamblea General de REBIUN en el 2004. Todavía vigente, la declaración insta a los autores a priorizar el envío de sus trabajos a aquellas publicaciones que sigan el modelo de acceso abierto y a no ceder totalmente sus derechos para poder depositar copia de sus trabajos en repositorios institucionales abiertos. También se insta a las instituciones académicas y a las autoridades políticas a promover el modelo mediante una serie de iniciativas.
En concreto, la Universidad Complutense de Madrid aprobó en Consejo de Gobierno de 27 de mayo de 2014 su política institucional de acceso abierto a la producción científica y académica (UCM, 2014). En la política se establece que:

Conforme a las recomendaciones de apoyo al acceso abierto a la información científica de la Comisión Europea, de la legislación estatal y de la legislación de la Comunidad Autónoma de Madrid, la UCM requiere el depósito de los trabajos de investigación de su personal docente e investigador.

El artículo 1 determina que:

El personal docente e investigador de la UCM depositará sus publicaciones, resultado de su actividad investigadora y académica (artículos científicos, libros o partes de libros, presentaciones en congresos, informes técnicos, etc.), en el repositorio institucional Complutense.

$Y$ en el artículo 8 se "recomienda que se evite la cesión total de los derechos de autor al publicar una obra, para permitir el depósito de la misma en repositorios de acceso abierto".

\section{Objetivos y metodología}

En el contexto descrito se inscribe la presente investigación, que tiene por finalidad conocer cuáles son las estrategias digitales de difusión y promoción de la investigación resultante de los proyectos de investigación concedidos a la Universidad Complutense de Madrid como entidad beneficiaria en el marco del Plan Estatal de investigación científico y técnica 2013-2016.

En última instancia, lo que nos interesa conocer es la actividad y práctica de los equipos de investigación en relación con la difusión de la información y producción científica resultado de los proyectos objeto de estudio.

Como objetivos específicos se plantean los siguientes:

- Calcular la producción científica total resultante de dichos proyectos.

- Identificar el porcentaje de la producción científica según el tipo de acceso: abierto o restringido.

- Determinar el cumplimiento de los requisitos sobre difusión en abierto de la Ley 14/2011, de 1 de junio, de la Ciencia, la Tecnología y la Innovación.

- Determinar el uso que hacen los equipos de los proyectos de investigación del repositorio institucional de la UCM, denominado E-prints Complutense, en relación con el depósito de la producción y la política institucional sobre acceso abierto. 
- Analizar qué porcentaje de producción científica respecto del total se puede recuperar desde un conjunto de plataformas digitales.

- Analizar la difusión de la investigación por ramas de conocimiento.

- Identificar la existencia de páginas web específicas dedicadas a los proyectos, así como el uso de la funcionalidad "proyectos" de la red social académica Research Gate (RG).

Para la delimitación de la muestra se establecieron una serie de criterios. Se seleccionó la modalidad 1 del Plan Estatal que corresponde a "Retos de Investigación" del programa orientado a los retos de la sociedad, en concreto la convocatoria del 2013. La elección de esa convocatoria supone que los proyectos tenían un alcance temporal de 3 años (2014-2016) y, por tanto, la mayor parte de la producción resultante ya está publicada, teniendo en cuenta también posibles periodos de embargo, y debería estar disponible en acceso abierto según los requisitos sobre difusión en abierto de la Ley 14/2011, de 1 de junio, de la Ciencia, la Tecnología y la Innovación y de la política institucional de la universidad.

Se consultó la resolución definitiva de concesión de ayudas y se seleccionaron los proyectos concedidos a la Universidad Complutense de Madrid como entidad beneficiaria (un total de 55 proyectos).

Se diseñó una tabla en Excel para la recogida de los datos y se crearon las fórmulas necesarias para el análisis y tratamiento de estos. En cuanto al recuento de la producción científica, se crearon campos numéricos con la aplicación de sumatorias. Para los indicadores "página web" y "proyecto en RG", destinados a conocer la existencia y uso de ambas herramientas, se crearon las siguientes variables: valor 1 , que manifiesta la existencia de tal indicador, y valor 0 , no existe.

Para el cálculo del total de la producción en el marco de los 55 proyectos, se seleccionaron aquellas plataformas digitales que permiten buscar y recuperar la producción científica por cada uno de los proyectos mediante la referencia o código de financiación: las bases de datos Web of Science y Scopus, el repositorio institucional de la UCM y el buscador Google Scholar.

Referente a la clasificación de la producción según el tipo de acceso tuvimos en cuenta las siguientes consideraciones:

- Acceso restringido: conformado por aquellos trabajos que solo se pueden obtener previo pago (toll access).

- Acceso abierto gratuito: sin barreras económicas, por tanto, los trabajos se pueden obtener y/o acceder de forma gratuita. Se ha descartado el acceso abierto libre, ya que no se ha analizado si los trabajos están libres o no de derechos de explotación o permisos de reutilización.

Para determinar la producción en acceso abierto se seleccionaron, por un lado, las dos vías más comúnmente reconocidas del acceso abierto: dorada y verde. Por otro lado, se contabilizaron también los trabajos depositados y accesibles por medio de otras herramientas como repositorios temáticos, redes sociales, páginas webs, portales bibliográficos, etc. y que han sido incluidos bajo el término otros recursos.

En consonancia con los objetivos de la investigación, para la vía verde se han considerado los trabajos depositados en el repositorio institucional de la Universidad Complutense de Madrid. Para el cálculo de documentos incluidos en la vía dorada se contabilizaron los así indicados por WoS y Scopus usando el filtro que ambas bases de datos tienen a tal efecto. Por último, para el conjunto de trabajos que están publicados y/o depositados en otros recursos, se contabilizaron los resultados arrojados por Google Scholar eliminando los duplicados, es decir, descartando aquellos trabajos que ya se habían computado para la vía verde y dorada.

En cuanto al análisis de la difusión de la investigación por ramas de conocimiento, se clasificaron los proyectos en cinco categorías generales, según la clasificación establecida en WoS: Artes y Humanidades, Ciencias de la Vida y Biomedicina, Ciencias Físicas, Ciencias Sociales y Tecnología.

En relación con la existencia de páginas web específicas y el uso de la característica "proyectos", se realizaron búsquedas libres en Google y ResearchGate mediante el título de los proyectos, tanto en español como en inglés, y por la referencia de financiación.

\section{Resultados}

\subsection{Análisis general}

Se han analizado un total de 55 proyectos pertenecientes a 19 centros de la Universidad Complutense de Madrid. En la tabla I se muestra la distribución de los proyectos concedidos a cada centro. Tras la aplicación de la metodología, se han recuperado un total de 429 documentos.

\begin{tabular}{lc}
\hline Centro & Proyectos \\
\hline Facultad de Veterinaria & 9 \\
\hline Facultad de Ciencias Químicas & 7 \\
\hline Facultad de Ciencias de la Información & 4 \\
\hline
\end{tabular}




\begin{tabular}{ll}
\hline Facultad de Ciencias Físicas & 4 \\
\hline Facultad de Ciencias Políticas y Sociología & 4 \\
\hline Facultad de Farmacia & 4 \\
\hline Facultad de Geografía e Historia & 4 \\
\hline Facultad de Informática & 3 \\
\hline Facultad de Ciencias Económicas y & 2 \\
\hline Empresariales & 2 \\
\hline Facultad de Ciencias Biológicas & 2 \\
\hline Facultad de Derecho & 2 \\
\hline Facultad de Filología & 2 \\
\hline Facultad de Medicina & 1 \\
\hline Facultad de Bellas Artes & 1 \\
\hline Facultad de Educación & 1 \\
\hline Facultad de Ciencias Geológicas & 1 \\
\hline Facultad de Ciencias Matemáticas & 1 \\
\hline Facultad de Filosofía & 1 \\
\hline Facultad de Óptica y Optometría &
\end{tabular}

Tabla I. Distribución de proyectos por centros

En relación con el total, el primero de los resultados que nos interesaba conocer era el número de documentos que son de acceso restringido frente al número de documentos que se encuentran en acceso abierto (Gráfico 1). En este caso, la situación podría considerarse como positiva pues un poco más de la mitad (55\%) de los trabajos se pueden consultar online y de forma gratuita, frente al $45 \%$ que requieren de pago para su consulta. Sin embargo, de este primer análisis se puede deducir que la muestra analizada no está cumpliendo con los requisitos nacionales de acceso abierto, pues todavía hay un porcentaje bastante elevado de trabajos que solo se pueden consultar previo pago.

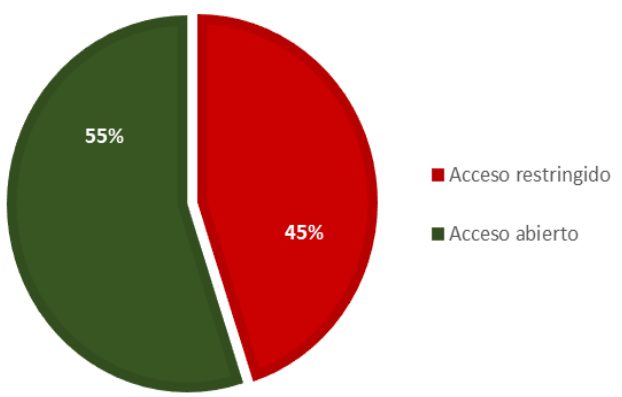

Gráfico 1. Trabajos en acceso abierto frente a acceso restringido

Para conocer el uso concedido al repositorio institucional de la UCM y los hábitos de los investigadores que forman parte de los proyectos seleccionados, se analizó también el porcentaje del total de los trabajos que han sido depositados en el repositorio institucional (Gráfico 2).

En esta ocasión, la situación es poco esperanzadora pues tan solo el $21 \%$ de la producción total resultante de los proyectos se encuentra depositada en el repositorio. Además, hay que tener en cuenta que el hecho de estar en el repositorio no significa que los trabajos sean de acceso libre. Del $21 \%$ mencionado, 7 de los trabajos son de acceso restringido, por lo que habría que solicitar permisos especiales para su consulta. Consecuentemente, podemos decir que los proyectos analizados no están cumpliendo con la política institucional de acceso abierto.

- Otros recursos - Repositorio UCM

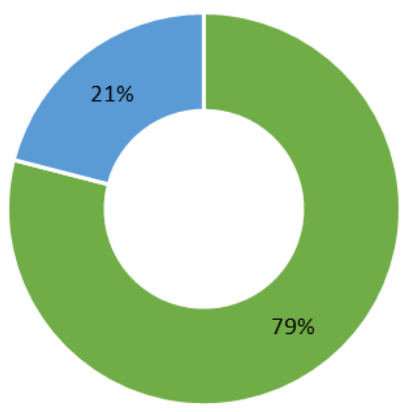

Gráfico 2. Producción depositada en el repositorio de la UCM

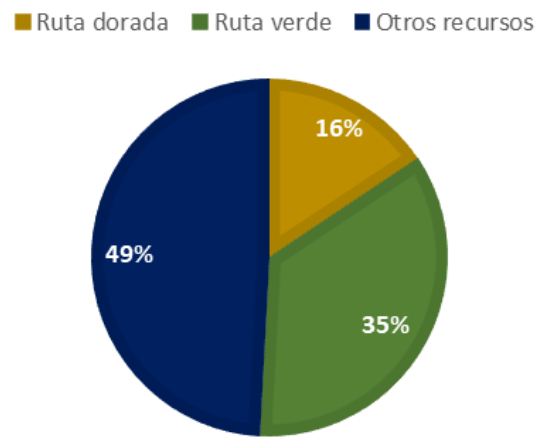

Gráfico 3. Formas de acceso abierto a la producción científica

En relación con la documentación que se encuentra en acceso abierto y haciendo una distinción entre ruta dorada $(16 \%)$, ruta verde $(35 \%)$ y otros recursos $(49 \%)$ que permiten el acceso online y gratuito a la producción científica, encontramos que casi la mitad de los trabajos están depositados en otros recursos (Gráfico 3). Este resultado es especialmente relevante teniendo en cuenta el marco normativo nacional e institucional sobre acceso abierto. Todos los trabajos que 
se pueden recuperar desde otras plataformas y consultar de forma gratuita, deberían estar depositados en el repositorio institucional.

Referente a las plataformas que permiten recuperar los trabajos por proyectos y que hemos seleccionado para la investigación, resulta interesante conocer cuál de ellas es la que arroja más resultados. Para ello, analizamos el porcentaje de documentos que se pueden recuperar en cada una de ellas del total de la producción en acceso abierto. En línea con los resultados de otras investigaciones, en este caso Google Scholar $(90 \%)$ también se configura como una herramienta muy potente para recuperar los trabajos relacionados con proyectos de investigación específicos (Gráfico 4).

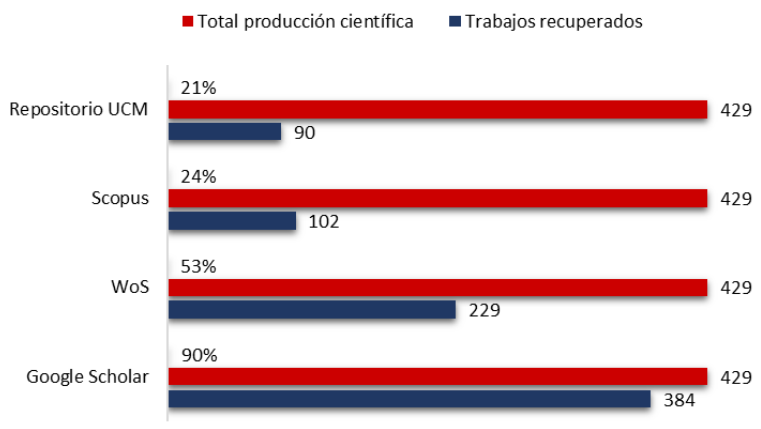

Gráfico 4. Recuperación de la producción científica por plataformas

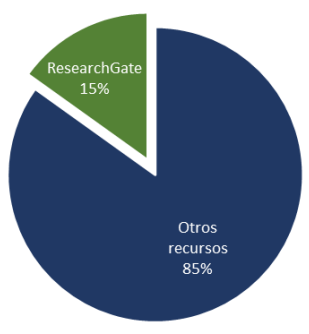

Gráfico 5. Producción científica disponible en ResearchGate

Dado que ResearchGate es en la actualidad una de las redes sociales académicas con más perfiles y más actividad en cuanto a promoción y visibilidad de la producción de los académicos e investigadores, se analizó el porcentaje de trabajos disponibles en la mencionada red social del total de la documentación disponible en acceso abierto (Gráfico 5). Se pone de manifiesto el poco uso que los investigadores de los proyectos analizados hacen de ResearchGate (15\%) para difundir su investigación. En concordancia con los resultados precedentes, es posible encontrar la producción científica en otros recursos (85\%) más que en ResearchGate.
Siguiendo con el análisis de las plataformas, analizamos el porcentaje de documentos que se pueden recuperar desde E-prints Complutense y Google Scholar del total de la producción en acceso abierto.

Como muestra el gráfico 6 , haciendo una búsqueda en el repositorio institucional podremos recuperar el $35 \%$ de los trabajos producidos en el marco de los proyectos de investigación, mientras que Google Scholar recupera el $65 \%$. De ese $65 \%$, el $47 \%$ corresponde a trabajos disponibles en otros recursos como repositorios temáticos, páginas web, portales bibliográficos, etc. mientras que el $18 \%$ de los trabajos son indizados desde ResearchGate.

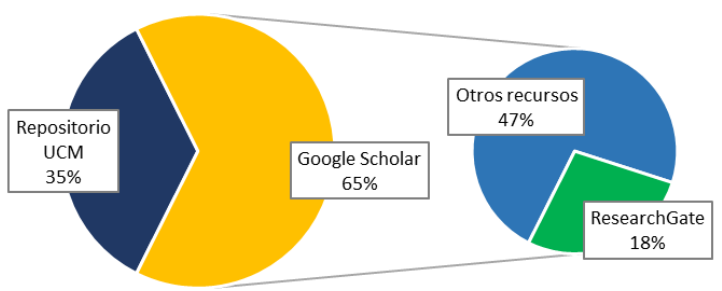

Gráfico 6. Porcentaje de producción científica recuperada por E-prints Complutense y Google Scholar

\subsection{Análisis por ramas de conocimiento}

En este apartado analizamos la publicación y recuperación de los resultados de investigación de los proyectos según una clasificación en 5 grandes ramas de conocimiento: Artes y Humanidades, Ciencias de la Vida y Biomedicina, Ciencias Físicas, Ciencias Sociales y Tecnología. Una vez clasificados los proyectos por objetos de estudio, obtuvimos el porcentaje de resultados de investigación para cada ámbito de conocimiento, generados por los proyectos durante su periodo de vigencia (Gráfico 7).

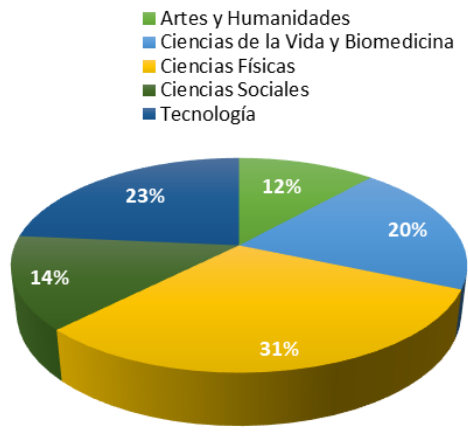

Gráfico 7. Producción científica por ramas de conocimiento 
Incluimos el gráfico 7 a modo informativo y para contextualizar los siguientes resultados. No se pretende juzgar la cantidad de producción científica, pues en última instancia depende de la naturaleza de cada área y de la cantidad de proyectos asignados a cada rama de conocimiento.

A continuación, se analiza, del total de la producción científica, en qué porcentaje la literatura es de acceso abierto o de acceso restringido (Gráfico 8). En este caso, se observa el predominio del acceso abierto en casi todas las ramas de conocimiento, a excepción de las Ciencias Físicas. Por otro lado, son las ramas de conocimiento de Artes y Humanidades y Ciencias Sociales las que cuentan con más producción en acceso abierto.

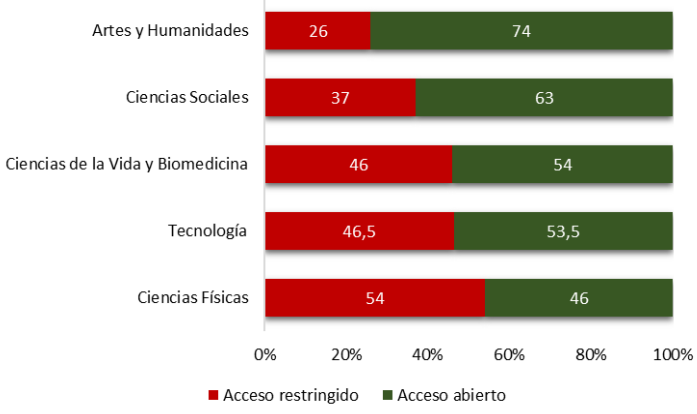

Gráfico 8. Investigación en acceso abierto y restringido por disciplinas

Resulta igualmente interesante conocer la importancia que se concede por ramas de conocimiento al depósito de los resultados de investigación de los proyectos en el repositorio institucional. Para ello, hemos analizado el porcentaje de publicaciones accesibles mediante el repositorio, del total de la producción científica (Gráfico 9).

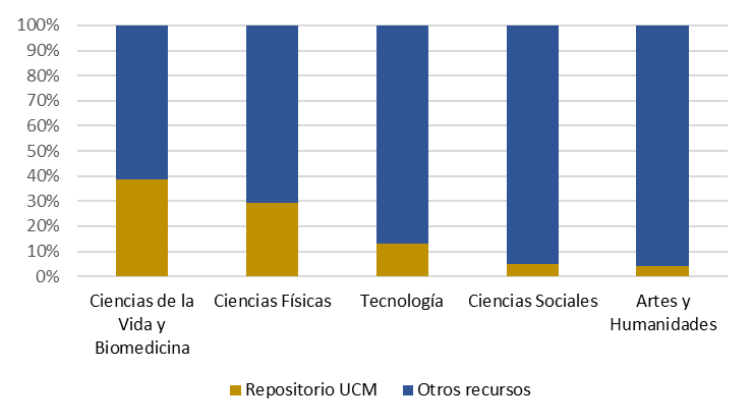

Gráfico 9. Producción depositada en el repositorio por ramas de conocimiento

Como se puede observar a simple vista en el gráfico 9, en ninguna de las ramas ni siquiera la mitad de la producción ha sido depositada en el repositorio. Sin embargo, cabe resaltar el caso de las Ciencias de la Vida y Biomedicina, y de las Ciencias Físicas, pues siendo dos áreas con muchas publicaciones de acceso restringido, son las que más trabajos tienen en el repositorio, con el $39 \%$ y el $29 \%$ respectivamente.

Por otro lado, a pesar de que Artes y Humanidades parece apostar por el acceso abierto, tan solo una mínima cantidad de los trabajos están disponibles en el repositorio (4\%).

Por último, se han analizado los diversos recursos en los que se encuentra la producción científica de acceso abierto, y en qué porcentaje encontramos trabajos en cada una de ellas (Gráfico 10).

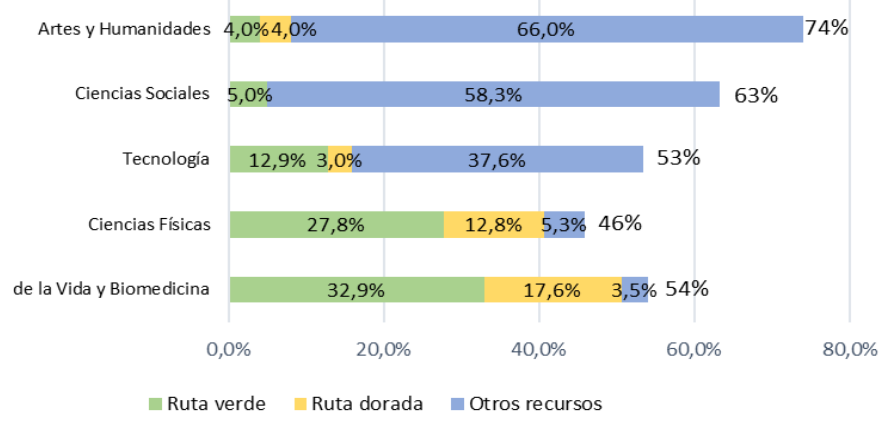

Gráfico 10. Formas de acceso abierto a la producción científica por ramas de conocimiento

En línea con los resultados precedentes, se puede observar que predomina la publicación en acceso abierto mediante otros recursos por encima del depósito en el repositorio institucional Complutense, siendo especialmente llamativo en Artes y Humanidades. Por el contrario, en el caso de las Ciencias Físicas, y Ciencias de la Vida y Biomedicina, gran parte de la producción en abierto está disponible en el repositorio.

\subsection{Existencia y análisis de páginas web}

Se evidencia que la principal vía de difusión de los investigadores que forman parte de los proyectos seleccionados es la publicación de resultados de investigación en revistas y monografías, siendo algunos accesibles gratuitamente mediante diversas plataformas en Internet. Esto produce una dispersión de la producción científica y dificulta el acceso y consulta a los trabajos desarrollados en el marco de un proyecto de investigación específico. Por este motivo, se considera de especial relevancia indagar en la existencia de páginas web dedicadas a los proyectos de investigación, donde se pueda consultar no solo la producción científica, sino que se detallen otros aspectos como los objetivos del proyecto, los miembros del equipo o las actividades que han llevado o están llevado a cabo. 
- Con página web = Información en Facultad/Dpto./Grupo - Sin página web

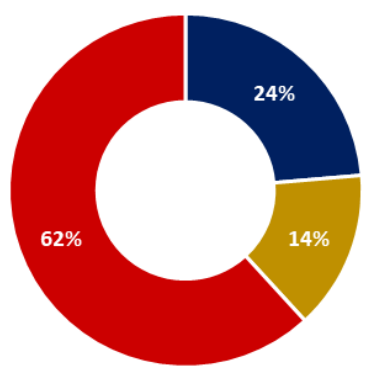

Gráfico 11. Proyectos con página web

El análisis de este indicador arroja resultados poco positivos, pues tan solo el $24 \%$ de los proyectos analizados cuenta con una página web específica con informaciones relativas a la actividad del proyecto (Gráfico 11). Del $14 \%$ de los proyectos se puede encontrar una referencia a los datos básicos del proyecto en las páginas web de las facultades, departamentos o grupos de investigación en los que se enmarca el proyecto. Este hecho constata, a su vez, que desde los centros tampoco se está promocionando la investigación que desarrolla su personal (Gráfico 11). El $62 \%$ restante corresponde a proyectos de los que no es posible localizar información alguna en Internet (Gráfico 11).

Aun disponiendo de página web, cabe mencionar que más de la mitad de dichas páginas solo proporciona información básica del proyecto: título, entidad financiadora, alcance temporal, investigador principal, descripción y palabras clave. Por lo tanto, realmente localizamos pocas páginas web, de las que se pueda extraer una información completa, profunda y actualizada sobre el proyecto, incluyendo actividades, publicaciones o patentes.

Asimismo, se realizó una búsqueda en ResearchGate, mediante el apartado "proyectos", para tratar de averiguar si los investigadores utilizan esta opción para dar a conocer su proyecto y resultados de investigación, así como comunicarse e interactuar con la comunidad científica. Tan solo 3 de los 55 proyectos $(5,5 \%)$ están presentes en ResearchGate.

\section{Conclusiones}

A la vista de los resultados, se evidencia que la principal vía de difusión de los resultados de investigación de los proyectos seleccionados es la publicación en revistas y monografías, siendo algunas accesibles gratuitamente mediante diversas plataformas en Internet.
Este hecho produce la dispersión de la producción científica y, por tanto, dificulta la recuperación y la consulta de los trabajos desarrollados en el marco de un proyecto de investigación específico.

La dispersión de la producción científica y la escasa existencia de páginas web dedicadas a los proyectos evidencia la ausencia de estrategias y planes de difusión digitales destinados a la promoción de las actividades y resultados de investigación en el marco de los proyectos.

Se detecta también la poca implicación y cumplimiento de los requisitos nacionales de acceso abierto y, particularmente, el incumplimiento de la política institucional sobre acceso abierto.

La falta de compromiso con el acceso abierto en el contexto analizado dificulta el desarrollo de estrategias y planes de difusión y promoción de la investigación en el entorno digital, repercutiendo directamente en la visibilidad y reputación académica de los investigadores, así como el impacto en la sociedad.

Sse constata que son necesarios esfuerzos por parte de todos los agentes implicados en el proceso de comunicación científica (universidades, centros de investigación, investigadores y entidades suscriptoras de revistas) para la plena implantación de la política nacional sobre acceso abierto. En línea con las recomendaciones de la FECYT, es fundamental la búsqueda de fórmulas basadas en incentivos para publicar en abierto, de forma que se considere como mérito en los procesos de evaluación, así como el uso de indicadores de citación alternativos. En relación con el apoyo a los investigadores, es fundamental la formación para la adquisición de hábitos, competencias y actitudes para publicar en abierto, así como familiarizarse con las prácticas de propiedad intelectual y cesión de derechos de explotación.

Las plataformas digitales, como las redes sociales académicas, las páginas web o los repositorios, se han consolidado como herramientas imprescindibles para la gestión de la información y la creación de planes de promoción. Sin embargo, se necesita una estrategia que haga efectiva la presencia y difusión de la actividad en el entorno digital.

En este sentido, es importante conocer las funcionalidades y características de cada uno de esos recursos digitales, para ser capaces de decidir cuáles integrar en los planes de difusión según los objetivos del proyecto y de los investigadores. No se considera un mejor plan aquel que abarca todas las opciones, sino el que se diseña 
con intención de aprovechar al máximo sus posibilidades.

La carencia de una estrategia de difusión de los resultados de investigación sin unos objetivos predefinidos produce la dispersión de la información relativa al proyecto, lo que dificulta la transferencia del conocimiento a las partes interesadas. En este sentido, contar con una página web puede ser un factor clave de diferenciación y éxito.

\section{Agradecimientos}

Al Ministerio de Educación, Cultura y Deporte (MECD) por el apoyo a la formación de jóvenes investigadores mediante el programa "Formación de profesorado universitario" (FPU).

\section{Referencias}

Abadal, E. (2004). Gestión de proyectos en información y documentación. Gijón: Ediciones Trea.

Abadal, E. (2014). El acceso abierto en Humanidades. Janus, 17-32. http://www.janusdigital.es/anexos/contribucion/descargar.htm?id=43

Baughman, M.S. (2015). Issue on the Transformation of Scholarly Communications // Research Library Issues. 287:1. http://publications.arl.org/rli287/1

Borrego, A. (2016). Measuring Compliance With a Spanish Government Open Access Mandate // Journal of the Association for Information Science and Technology. 67:4 (2016) 757-764. https://doi.org/10.1002/asi.23422

Budapest Open Access Initiative (2002). http://www.budapestopenaccessinitiative.org (2018-04-01).

Campos-Freire, F.; Rúas-Araújo, J. (2016). Uso de las redes sociales digitales profesionales y científicas: el caso de las 3 universidades gallegas // El profesional de la información. 3:25 (2016) 431-440. https://recyt.fecyt.es/index.php/EPI/article/view/epi.2016.may.13

CRUE (2004). Declaración de la CRUE en apoyo del modelo de acceso electrónico abierto. http://biblioteca.ucm.es/boletin/bibliotecario/01/acceso.pdf (201804-01).

España (2001). Ley Orgánica 6/2001, de 21 de diciembre, de Universidades // Boletín Oficial del Estado. 307 (24 diciembre de 2001) 49400-49425. https://www.boe.es/boe /dias/2001/12/24/pdfs/A49400-49425.pdf

España (2011). Ley 14/2011, de 1 de junio, de la Ciencia, la Tecnología y la Innovación // Boletín Oficial del Estado. 131 (2 de junio de 2011) 54387-54455. https://www.boe. es/boe/dias/2011/06/02/pdfs/BOE-A-2011-9617.pdf

España (2013). Orden ECC/1780/2013, de 30 de septiembre, por la que se aprueban las bases reguladoras para la concesión de ayudas públicas del Programa Estatal de Investigación, Desarrollo e Innovación Orientada a los Retos de la Sociedad, en el marco del Plan Estatal de Investigación Científica y Técnica y de Innovación 2013-2016 // Boletín Oficial del Estado. 236 (2 de octubre de 2013) 80222-80256. http://boe.es/boe/dias/2013/10/02/pdfs/ BOE-A-2013-10259.pdf

FECYT (2016). Informe de la comisión de seguimiento sobre el grado de cumplimiento del artículo 37 de la Ley de Ciencia. https://www.recolecta.fecyt.es/sites/default/files/contenido/documentos/CumplimientoOA.pdf (201804-01).
Fundación Botín (2016). Guía 1: explotación y difusión de resultados. https://www.fundacionbotin.org/89dguuytdfr276 ed uploads/BIBLIOTECA\%20Y\%20PUBLICACIONES/ Guia1_Explotacion_difusion_resultados_Enlaces.pdf (2018-04-01).

García-Peñalvo, F. J. (2017). Identidad digital del doctorando. Salamanca: Grupo GRIAL. https://repositorio.grial.eu/handle/grial/821

González-Díaz, C.; Iglesias-García, M.; Codina, L. (2015). Presencia de las universidades españolas en las redes sociales digitales científicas: caso de los estudios de comunicación // El profesional de la información. 5:24 (2015) 640-647. https://recyt.fecyt.es/index.php/EPI/article/view/epi.2015.sep.12

López Yepes, J. (2010). Cómo se hace una tesis: trabajos de fin de grado, máster y tesis doctorales. México: Library Outsourcing Service.

López-Pérez, L.; Olvera-Lobo, M. D. (2016). Comunicación pública de la ciencia a través de la web 2.0. El caso de los centros de investigación y universidades públicas de España // El profesional de la información. 3:25 (2016) 441-448. https://recyt.fecyt.es/index.php/EPI/article/view/ epi.2016.may. 14

MINECO (2014). Guía del participante: Horizonte2020. http://eshorizonte2020.cdti.es/recursos/doc/Programas/ Cooperacion_internacional/HORIZONTE\%202020/292 36_2872872014135311.pdf (2018-04-01).

MINECO (2017). Plan Estatal Investigación Científica y Técnica y de Innovación 2017-2020. http://www.idi.mineco.gob.es/stfls/MICINN/Prensa/FICHEROS/2018/PlanEstatallDI.pdf

Ollé, C.; Abadal, E. (2013). La edición universitaria en el contexto de la ciencia abierta. Barcelona: Editorial UOC.

Parlamento Europeo (2013). Reglamento (UE) no 1291/2013 del Parlamento Europeo y del Consejo de 11 de diciembre de 2013 por el que se establece Horizonte 2020, Programa Marco de Investigación e Innovación (2014-2020) y por el que se deroga la Decisión n¹982/2006/CE. http://ec.europa.eu/research/participants/data/ref/h2020/ legal_basis/fp/h2020-eu-establact_es.pdf

Sanz Menéndez, L. (2005). Universidad e investigación: la financiación competitiva de los proyectos de I+D, con especial referencia a las Ciencias Sociales y Económicas // Reis. 109:05 (2005) 181-218. http://www.reis.cis.es/ REIS/PDF/REIS_109_081168259779178.pdf

Sawant, S. (2012). Transformation of the scholarly communication cycle // Library Hi Tech News. 29:10 (2012) 21-24. http://eprints.rclis.org/18894/

Seguí Simarro, J.M.; Poza Luján, J.L.; Mulet Salort, J.M. (2015). Estrategias de divulgación científica. Valencia, Editorial de la Universidad Politécnica de Valencia.

Siso Calvo, B.; Arquero Avilés, R. (2017). Personal branding en investigación: prospección y retos en el área de Ciencias de la Documentación // Actas de las I Jornadas en Ciencias de la Documentación. Madrid, mayo 2017. http://eprints.ucm.es/45679/1/Personal\%20branding\%20en\%20investigaci\%C3\%B3n_Siso_Arquero.pdf

Suber, P. (2012). Open access. Cambridge: The MIT Press Suber, P. (2015). Acceso abierto. [S.I.]: CLACSO.

UCM (2014). Política institucional de acceso abierto a la producción científica y académica de la UCM. https://www. ucm.es/repositoriodigital/acceso-abierto-ucm (2018-0401).

Enviado: 2018-04-01. Segunda versión: 2018-05-29. Aceptado: 2018-09-05 